\section{NÍVEL DE ATIVIDADE FÍSICA E SÍNDROME LIPODISTRÓFICA EM PACIENTES COM HIV/AIDS}

\author{
LEVEL OF PHYSICAL ACTIVITY AND LIPODYSTROPHY SYNDROME IN PATIENTS WITH HIVIAIDS \\ NIVEL DE ACTIVIDAD FISICA Y SÍNDROME DE LIPODISTROFIA EN PACIENTES CON VIH/SIDA
}

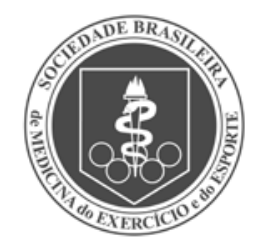

Artigo Original

Original ARTICLE

Artículo Original
Luiz Fernando Gouvêa-e-Silva ${ }^{1,2}$ (Educador Físico)

Renato do Carmo Said ${ }^{2}$ (Médico)

Kátia Simone Kietzer² (Fisioterapeuta)

Jofre Jacob da Silva Freitas ${ }^{2}$ (Biomédico)

Marília Brasil Xavier1,2

(Médica)

1. Universidade Federal do Pará, Programa de Pós-graduação em Doenças Tropicais do Núcleo de Medicina Tropical, Belém, Pará, Brasil. 2. Universidade do Estado do Pará, Centro de Ciências Biológicas e da Saúde, Santarém, Pará, Brasil.

\section{Correspondência:}

Av. Plácido de Castro, 1399, Bairro Aparecida, Santarém, PA, Brasil. 68040-090.

Ifgouvea@yahoo.com.br

\section{RESUMO}

Introdução: A atividade física pode ser uma aliada para minimizar o surgimento da lipodistrofia e seus efeitos deletérios. Objetivo: Analisar a relação do nível de atividade física (NAF) e a síndrome lipodistrófica com a composição corporal e a qualidade de vida (QV) de portadores do HIV/Aids. Métodos: Participaram do estudo 120 pacientes com HIV/Aids (38,55 $\pm 11,47$ anos). Foram avaliados quanto ao índice de massa corporal, composição corporal, taxa metabólica basal, índice cintura-quadril, QV, presença, tipo e local de lipodistrofia, força de preensão manual e NAF. Resultados: Constatou-se que 70\% eram sedentários; além disso, a massa magra foi 9,5\% maior nos pacientes ativos, bem como a força muscular de preensão com a mão direita (11\%) e esquerda (12\%) e a taxa metabólica basal (6,9\%). Quanto à associação da QV ao NAF, foi notada correlação fraca apenas para a preocupação com o sigilo $(r=0,2160 ; p=0,0210)$, bem como, os pacientes ativos demonstraram melhor resultado que os sedentários $(p<0,05)$. Dos pacientes que apresentaram lipodistrofia, $66 \%$ eram sedentários; o tipo predominante foi a lipodistrofia mista (46\%) e as regiões mais comuns foram a face e abdome. Ressalta-se que nos pacientes sem a síndrome a taxa metabólica basal foi maior $(p=0,0175)$ e o tempo de infecção $(p=0,0020)$ e de TARV $(p=0,0002)$ foram menores. Conclusão: Conclui-se, conforme metodologia adotada, que o sedentarismo predominou, o NAF apresentou associação positiva somente com a preocupação com o sigilo e os sedentários apresentaram dois domínios abaixo do escore de 50 (preocupação financeira e com o sigilo), com relação a um domínio dos pacientes ativos. A lipodistrofia esteve presente em $37 \%$ da amostra, sendo o tipo mais presente a lipodistrofia mista, e os locais de maior ocorrência foram a face e o abdome. Para esse grupo o NAF não se apresentou positivo para a composição corporal e a presença da lipodistrofia não influenciou na QV.

Descritores: lipodistrofia; atividade motora; HIV; síndrome de imunodeficiência adquirida.

\section{ABSTRACT}

Introduction: Physical activity can be an ally to minimize the appearance of lipodystrophy and its deleterious effects. Objective: To analyze the relationship between physical activity level (PAL) and lipodystrophy syndrome with body composition and quality of life (QOL) of patients with HIV/Aids. Methods: The study included 120 patients with HIV/Aids (38.55 \pm 11.47 years). They were evaluated for body mass index, body composition, basal metabolic rate, waist-hip ratio, QOL, presence, type, and location of lipodystrophy, hand grip strength, and PAL. Results: It was found that 70\% were sedentary; in addition, lean body mass was $9.5 \%$ higher in active patients, as well as muscle strength of right hand grip (11\%) and left hand grip (12\%) and basal metabolic rate (6.9\%). Regarding the association between $\mathrm{QOL}$ and PAL, a weak correlation was noted only in relation to confidentiality $(r=0.2160, p=0.0210)$, and active patients showed better results than sedentary ones $(p<0.05)$. Of the patients with lipodystrophy, 66\% were sedentary; the predominant type was mixed lipodystrophy (46\%) and the most common regions were face and abdomen. It is noteworthy that in patients without the syndrome, the basal metabolic rate was higher $(p=0.0175)$ and the time of infection $(p=0.0020)$ and HAART $(p=0.0002)$ were lower. Conclusion: We conclude, according to the methodology, that sedentary life was predominant, PAL showed positive association only with the concern with confidentiality and the sedentary patients showed two domains below the score of 50 (financial concern and confidentiality), in relation to one domain of active patients. Lipodystrophy was present in $37 \%$ of the sample, being the most prevalent type the mixed lipodystrophy, and the main sites of occurrence were face and abdomen. For this group PAL was not positive for body composition and the presence of lipodystrophy did not influence QOL.

Keywords: lipodystrophy; motor activity; HIV; acquired immunodeficiency syndrome.

\section{RESUMEN}

Introducción: La actividad física puede ser un aliado para minimizar la aparición de lipodistrofia y sus efectos nocivos. Objetivo: Analizar la relación entre el nivel de actividad física (NAF) y el síndrome de lipodistrofia con la composición corporal y la calidad de vida (CV) de las personas con VIH/Sida. Métodos: Se incluyó 120 pacientes con VIH/SIDA (38,55 $\pm 11,47$ años). Ellos fueron evaluados en relación al índice de masa corporal, la composición corporal, metabolismo basal, el índice cintura-cadera, CV, presencia, tipo y ubicación de lipodistrofia, fuerza de prensión 
manual y NAF. Resultados: Se encontró que el 70\% eran sedentarios; además, la masa magra fue 9,5\% mayor en los pacientes activos, asi como la fuerza muscular de prensión manual derecha (11\%) y prensión manual izquierda (12\%) y la tasa metabólica basal (6,9\%). En cuanto a la asociación de la calidad de vida y NAF, se observó correlación débil solamente con la preocupación con la confidencialidad ( $r=0,2160 ; p=0,0210)$, así como los pacientes activos han demostrado mejores resultados que los sedentarios $(p<0,05)$. De los pacientes con lipodistrofia, $66 \%$ eran sedentarios, el tipo predominante fue lipodistrofia mixta (46\%) y las regiones más afectadas fueron la cara y el abdomen. Es de destacar que en pacientes sin sindrome, la tasa metabólica basal fue mayor $(p=0,0175)$ y el tiempo de infección $(p=0,0020)$ y TARV $(p=0,0002)$ fueron inferiores. Conclusión: Se concluye que, de acuerdo con la metodología adoptada, el sedentarismo es predominante, el NAF presentó una asociación positiva sólo con la preocupación por la confidencialidad y los pacientes sedentarios tenían dos dominios por debajo de la puntuación de 50 (preocupación financiera y confidencialidad), con respecto a un dominio de los pacientes activos. La lipodistrofia estuvo presente en 37\% de la muestra, siendo el tipo más presente la lipodistrofia mixta y los sitios de mayor incidencia la cara y el abdomen. Para este grupo la NAF no se ha presentado positiva para la composición corporal y la presencia de lipodistrofia no influyó en la calidad de vida.

Descriptores: lipodistrofia; actividad motora; VIH, síndrome de inmunodeficiencia adquirida.

\section{INTRODUÇÃO}

A Síndrome da Imunodeficiência Adquirida - Aids é uma patologia que ocasiona imunossupressão crônica, considerada uma epidemia mundial. No ano de 2009 foram apontados 13.661 novos casos, dos mais de 544.000 diagnosticados de 1980 a 2009. Sendo que a região sudeste acumula o maior número de casos, 323.069 casos, e a região norte fica em $5^{\circ}$ lugar, com 21.389 casos diagnosticados neste intervalo de tempo. Contudo, o estado do Pará apresenta o maior número de casos, 9.428, em relação aos outros estados da região norte'. Já para junho de 2012 foram notificados 17.819 novos casos, dos mais de 656.700 casos diagnosticados de 1980 a junho de 2012. A região sudeste continua com o maior número, 367.540 casos, e a região norte mantém o $5^{\circ}$ lugar com 32.140 casos, um aumento de $50.26 \%$ em dois anos e meio. O estado do Pará, por sua vez, aumentou $48.47 \%$ o número de diagnósticos (13.998 casos)².

Para minimizar os efeitos ocasionados pela imunossupressão, em 1990, a terapia antirretroviral altamente ativa (TARV) foi incorporada ao tratamento. Essa introdução medicamentosa melhorou a qualidade de vida e aumentou a duração da vida destes pacientes, ou seja, nota-se que a expectativa de vida em 4-5 anos aumentou de 76,5\% em 2002 para 79\% em 2004'. No ano de 2012, 313 mil pacientes faziam uso da TARV 3 . Um ponto negativo da TARV é a indução da Síndrome Lipodistrófica, ou seja, alterações na distribuição de gordura corporal e anormalidades metabólicas, que elevam o risco para o desenvolvimento de doenças cardiovasculares 4,5 .

Como meio de atenuar esses efeitos destacar-se a prática da atividade física, em que a mesma pode contribuir na melhora da autoestima $a^{6,7}$, da força muscular ${ }^{8,9}$, do sistema imune ${ }^{10}$, dos marcadores bioquímicos, da massa muscular ${ }^{8,11,12}$, do sistema cardiorrespiratório ${ }^{13} \mathrm{e}$ do perfil antropométric ${ }^{14,15}$.

Desta forma, o objetivo deste manuscrito foi analisar a relação do nível de atividade física e da Síndrome Lipodistrófica com a composição corporal e a qualidade de vida de portadores do HIV/Aids.

\section{MATERIAIS E MÉTODOS}

A amostra foi composta por 120 pacientes do Centro de Testagem e Aconselhamento - CTA do Serviço de Assistência Especializado - SAE do município de Santarém - Pará. A média de idade dos pacientes foi de $38.55 \pm 11.47$ anos, com $65 \%$ pertencente ao gênero masculino $(n=78)$.

Os critérios de inclusão adotados foram idade entre 18 e 70 anos, com utilização ou não da TARV e dar o consentimento através da assinatura do Termo de Consentimento Livre e Esclarecido. Já como critérios de exclusão foram observados a utilização crônica de glicocorticoides, estar em estado gestacional, possuir marca-passo, ser portador de tumores malignos e doença mental diagnosticados.

O estudo foi aprovado pelo Comitê de Ética em Pesquisa do Núcleo de Medicina Tropical da Universidade Federal do Pará, sob o CAAE: 27162514.9.0000.5172.

Para a realização das medidas da massa corporal utilizou-se uma balança analógica (Welmy Indústria Comércio, Santa Bárbara D'Oeste - SP, Brasil), e para a estatura o estadiômetro acoplado à mesma. Para as circunferências foi empregada uma fita antropométrica (Sanny, São Paulo - SP, Brasil). O índice de massa corporal foi calculado dividindo-se a massa corporal pela estatura ao quadrado e a índice cintura-quadril ICQ pela divisão dos valores das circunferências do abdome pelo quadril.

A composição corporal foi aferida com a utilização do aparelho de bioimpedância tetrapolar (Maltron International Ltd, modelo BF 900, Rayleigh, Essex, Inglaterra), onde foram fornecidos os dados referentes ao percentual de gordura, massa magra, massa gorda e a taxa metabólica basal.

O somatório das dobras cutâneas foi encontrado através da aferição e soma das dobras do bíceps, tríceps, subescapular, abdominal, supra-ilíaca, coxa e panturrilha medial, utilizando-se as técnicas de Fernandes Filho' ${ }^{16}$, com um adipômetro de precisão de $0,1 \mathrm{~mm}$ (Cescorf Equipamentos para Esporte Ltda, Porto Alegre - RS, Brasil). Para realizar a classificação da Síndrome Lipodistrófica" ${ }^{17}$ foi utilizado os seguintes parâmetros:

a. Lipoatrofia: no exame físico foi observado a presença de lipoatrofia facial (definhamento da face), periférica (membros) e nas nádegas, podendo resultar em proeminência relativa de musculatura e circulação venosa;

b. Lipohipertrofia: no exame físico foram notadas as características de lipohipertrofia abdominal (volume abdominal aumentado), ginecomastia nos homens e aumento das mamas nas mulheres, lipohipertrofia dorso-cervical (pescoço de búfalo/gibosidade dorsal) e acúmulos de gorduras em outros locais como as regiões submentoniana e pubiana;

c. Mista: associação de lipoatrofia e lipohipertrofia.

A força muscular de preensão manual foi aferida por meio de um dinamômetro (Kratos Equipamentos Industriais, Modelo ZM, Cotia - SP, Brasil) (precisão de um Kg), onde o paciente ficou em posição ortostática, com o antebraço que empunhava o aparelho em um ângulo de $90^{\circ}$ com o braço e a mão em uma posição semipronada. 
Para avaliar o nível de atividade física, este estudo optou por utilizar o questionário IPAQ - versão curta (Questionário Internacional de Atividade Física) por ser um instrumento de fácil aplicação e de baixo custo ${ }^{18}$.

O questionário contém perguntas relacionadas à frequência (dia por semana) e duração (tempo por dia) da realização de atividades físicas de forma moderada, vigorosa e caminhada. A classificação final é dada como a pessoa sendo sedentária, insuficientemente ativo um ou dois, ativa e muito ativa. Para melhor demonstrar e relacionar os resultados deste estudo considerou-se apenas duas classificações, sedentário e ativo, onde a primeira envolve as classificações de sedentário e as de insuficientemente ativos e a segunda agrupou os pacientes classificados como ativo e muito ativo.

Para verificar a qualidade de vida dos pacientes foi utilizado o questionário de qualidade de vida HAT-QoL (HIV/Aids - Targeted Quality of Life) que apresenta nove domínios: função geral (seis perguntas), satisfação com a vida (quatro perguntas), preocupações com a saúde (quatro perguntas), preocupações financeiras (três perguntas), preocupação com a medicação (cinco perguntas), aceitação do HIV (duas perguntas), preocupação com o sigilo (cinco perguntas), confiança profissional (três perguntas) e função sexual (duas perguntas). Ressalta-se que o mesmo foi traduzido e validado para o Brasil $^{19}$.

\section{Análise dos dados}

Primeiramente foi verificada a normalidade dos dados e para os dados paramétricos foi utilizado o Teste T não pareado e para os dados não paramétricos o Teste Mann-Whitney para comparar os dados entre os grupos analisados. Para correlacionar o nível de atividade física (sedentário, insuficientemente ativo, ativo e muito ativo) com os domínios da qualidade de vida foi empregado a Correlação de Spearman. Para os testes descritos até então foi usado o Software Graphpad Prism 3.0. Para verificar a associação entre o nível de atividade física e o tipo e localização da lipodistrofia foram utilizados os Teste Qui-Quadrado e de Contingência C, através do Software BioEstat 5.2. Para todas as análises o nível de significância adotado foi de $\mathrm{p}<0.05$.

\section{RESULTADOS}

Com a realização da análise dos dados pôde-se notar que não ocorreram diferenças estatísticas para as variáveis comparadas entre os pacientes classificados como sedentários dos ativos (Tabela 1), visto que os pacientes sedentários representaram $70 \%(n=84)$ e os ativos 30\% $(n=36)$. Contudo ressalta-se que a massa magra foi 9,5\% maior nos pacientes ativos, bem como, a força muscular de preensão com a mão direita (11\%) e esquerda (12\%), a taxa metabólica basal (6,9\%), o tempo de infecção com o HIV $(37,7 \%)$ e da terapia antirretroviral $(48,9 \%)$.

Para realizar a descrição dos resultados da qualidade de vida (Tabela 2), adotar-se-á que índices até 49 são ruins, de 50 a 79 são satisfatórios e de 80 a 100 são bons. Desta forma, pode-se descrever que para os pacientes classificados como sedentários a preocupação financeira (40.84) e com o sigilo (29,62), foram os domínios mais preocupantes, pois apresentaram os menores índices. Já nos ativos apenas a preocupação com o sigilo $(44,86)$ foi ruim e a preocupação financeira foi satisfatória $(53,65)$.

Como satisfatório nota-se os domínios da função geral (62,88 vs 70,48), satisfação com a vida $(70,90$ vs 73,37), preocupação com a saúde $(55,25$ vs 64,17), preocupação com a medicação $(73,33$ vs 76,82), aceitação do HIV $(50,96$ vs 56,72) e função sexual $(69,65$ vs 73,03), para os sedentários e ativos, respectivamente. Apenas o domínio da confiança profissional apresentou boa pontuação para os sedentários $(89,67)$ e ativos $(86,58)$.
Tabela 1. Demonstrativo dos valores antropométricos, da composição corporal, força de preensão manual, tempo de infecção e terapia antirretroviral em sedentários e ativos.

\begin{tabular}{|c|c|c|c|c|c|c|c|c|c|}
\hline & \multicolumn{4}{|c|}{ Sedentário } & \multicolumn{4}{|c|}{ Ativo } & \multirow[b]{2}{*}{ p } \\
\hline & mín & máx & média & $\mathrm{dp}$ & mín & máx & média & $d p$ & \\
\hline$M C(\mathrm{Kg})$ & 39,00 & 97 & 63,04 & 11,89 & 48,60 & 88 & 67,79 & 9,91 & $>0,05$ \\
\hline $\mathrm{EST}(\mathrm{m})$ & 1,42 & 1,82 & 1,62 & 0,09 & 1,45 & 1,87 & 1,65 & 0,09 & $>0,05$ \\
\hline $\operatorname{IMC}\left(\mathrm{Kg} / \mathrm{m}^{2}\right)$ & 15,82 & 36,03 & 24,13 & 3,91 & 19,82 & 35,80 & 25,04 & 3,51 & $>0,05$ \\
\hline ICQ & 0,57 & 1,08 & 0,92 & 0,08 & 0,80 & 1,07 & 0,94 & 0,07 & $>0,05$ \\
\hline$\Sigma \mathrm{DC}(\mathrm{mm})$ & 27,30 & 294,3 & 109,49 & 53,05 & 31,70 & 202,9 & 104,65 & 43,61 & $>0,05$ \\
\hline$\% G$ & 4,4 & 50,7 & 24,79 & 9,53 & 8,5 & 45,7 & 24,03 & 9,36 & $>0,05$ \\
\hline MM (Kg) & 30,89 & 79,65 & 46,95 & 8,59 & 31,69 & 73,22 & 51,39 & 9,12 & $>0,05$ \\
\hline$M G(K g)$ & 1,78 & 45,02 & 16,10 & 8,02 & 5,1 & 39,3 & 16,40 & 7,34 & $>0,05$ \\
\hline TMB (kcal) & 1083 & 2016 & 1448,40 & 197,23 & 1115 & 1978 & 1548,67 & 198,90 & $>0,05$ \\
\hline FMd (Kg) & 10 & 53 & 31,07 & 8,88 & 20 & 51 & 34,53 & 9,14 & $>0,05$ \\
\hline FMe (Kg) & 15 & 58 & 31,37 & 9,34 & 17 & 52 & 35,15 & 10,14 & $>0,05$ \\
\hline Tinf (meses) & 1 & 183 & 42,99 & 36,71 & 3 & 180 & 59,19 & 50,84 & $>0,05$ \\
\hline $\begin{array}{l}\text { TTARV } \\
\text { (meses) }\end{array}$ & 0 & 146 & 34,30 & 33,82 & 0 & 180 & 51,08 & 50,80 & $>0,05$ \\
\hline
\end{tabular}

MC - massa corporal; EST - estatura; IMC - índice de massa corporal; ICQ - índice cintura-quadril; EDC - somatório das dobras cutâneas; $\%$ - percentual de gordura; MM - massa magra; MG - massa gorda; TMB - taxa metabólic basal; FMd - força muscular de apreensão da mão direita; FMe - força de apreensão da mão esquerda; Tinf - tempo de infecção pelo HIV; TTARV - tempo de terapia antirretroviral; mín - mínimo; máx - máximo; dp - desvio padrão.

Tabela 2. Demonstrativo dos valores da qualidade de vida em sedentários e ativos

\begin{tabular}{|c|c|c|c|c|c|c|c|c|c|c|}
\hline & \multicolumn{4}{|c|}{ Sedentário } & \multicolumn{4}{|c|}{ Ativo } & \multirow[b]{2}{*}{1} & \multirow[b]{2}{*}{$p$} \\
\hline & mín & máx & média & $d p$ & mín & máx & média & $d p$ & & \\
\hline $\mathrm{FG}$ & 4 & 100 & 62,88 & 22,72 & 33 & 100 & 70,48 & 17,19 & 0,1630 & $>0,05$ \\
\hline SV & 6 & 100 & 70,90 & 23,75 & 25 & 100 & 73,37 & 17,98 & 0,1096 & $>0,05$ \\
\hline PS & 0 & 100 & 55,25 & 30,97 & 0 & 100 & 64,17 & 33,35 & 0,1330 & $>0,05$ \\
\hline $\mathrm{PF}$ & 0 & 100 & 40,84 & 33,46 & 0 & 100 & 53,65 & 30,01 & 0,1827 & $>0,05$ \\
\hline PM & 20 & 100 & 73,33 & 22,96 & 30 & 100 & 76,82 & 22,14 & 0,0213 & $>0,05$ \\
\hline AHIV & 0 & 100 & 50,96 & 33,48 & 0 & 100 & 56,72 & 40,20 & 0,1172 & $>0,05$ \\
\hline Psig & 0 & 100 & $29,62^{*}$ & 26,01 & 0 & 100 & 44,86 & 29,60 & 0,2160 & 0,0210 \\
\hline$C P$ & 42 & 100 & 89,67 & 16,15 & 17 & 100 & 86,58 & 23,46 & $-0,0455$ & $>0,05$ \\
\hline FX & 0 & 100 & 69,65 & 36,16 & 0 & 100 & 73,03 & 32,33 & $-0,0222$ & $>0,05$ \\
\hline
\end{tabular}

G - função geral; SV - satisfação com a vida; PS - preocupação com a saúde, PF - preocupação financeir ; PM - preocupaçăo com a medicaçăo; AHI - aceitaçăo com o HIV; Psig - preocupaçăo com o sigilo; CP - confiança no profissional; FX - função sexual; mín - mínimo; máx - máximo; dp - desvio padrão; $r$ - correlação de Spearman do nivel de atividade física com as dimensões da qualidade de vida. *Diferença estatística dos participantes ativos $-\mathrm{p}<0.05$

Observa-se que os índices para os domínios da qualidade de vida foram superiores para os pacientes ativos, exceção para a confiança profissional, contudo não estatística essa diferença, exceção para a preocupação com o sigilo ( $p=0,0039)$.

Analisando a correlação do nível de atividade física com os domínios da qualidade de vida, foi observada correlação positiva fraca apenas para a preocupação com o sigilo ( $r=0,2160 ; p=0,0210)$.

Observando-se a presença de lipodistrofia nos pacientes (Tabela 3), nota-se que 37\% ( $n=44)$ da amostra apresenta algum tipo de lipodistrofia, sendo que a tipo mista foi a mais presente (46\%), seguida pela lipoatrofia (34\%) e lipohipertrofia (20\%). Quanto à região, observou-se 
Tabela 3. Demonstrativo da caracterização da Síndrome Lipodistrófica em sedentários e ativos.

\begin{tabular}{c|c|c|c|c}
\hline & Sedentário & Ativo & $\mathbf{n}$ & $\%$ \\
\hline Lipoatrofia & 11 & 4 & 15 & 34 \\
\hline Lipohipertrofia & 6 & 3 & 9 & 20 \\
\hline Mista & 12 & 8 & 20 & 46 \\
\hline $\mathrm{n}$ & 29 & 15 & 44 & 100 \\
\hline$\%$ & 66 & 34 & & \\
\hline \multicolumn{5}{c|}{ Locais com apresentação de Lipoatrofia } \\
\hline Face & 19 & 5 & 24 & 56 \\
\hline MS & 8 & 4 & 12 & 27 \\
\hline Nádega & 9 & 4 & 13 & 30 \\
\hline \multicolumn{5}{|c|}{ Locais com apresentação de Lipohipertrofia } \\
\hline Abdome & 16 & 8 & 24 & 56 \\
\hline Dorsocervical & 8 & 4 & 12 & 27 \\
\hline Ginecomastia & 4 & 1 & 5 & 11 \\
\hline $\mathrm{n}$ & 73 & 33 & 106 & 100 \\
\hline \% & 69 & 31 & & 36 \\
\hline
\end{tabular}

n - número de identificações; \% - percentual; MS - membro superior; MI - membro inferior.

que a lipoatrofia facial (56\%) e lipohipertrofia abominal (56\%) foram as mais presentes, seguidas pela lipoatrofia das nádegas (36\%), dos membros inferiores (30\%), membros superiores (27\%) e lipohipertrofia dorsocervical (27\%) e ginecomastia (11\%).

Analisando a associação entre o nível de atividade física e o tipo ou região da lipodistrofia não foi observada nenhuma relação estatística. Já dos pacientes que demonstraram lipodistrofia, 66\% eram sedentários e 34\% ativos, contudo esteve mais presente nos ativos $(41,67 \%)$. Quanto à região, observa-se que os sedentários demonstraram 73\% das modificações, sendo elas mais presente na face (19\%) e abdome (16\%). Já nos ativos, as regiões mais destacadas foram o abdome (8\%) e as nádegas (7\%).

Verificando a diferença entre os pacientes com Síndrome Lipodistrófica e sem a Síndrome (Tabela 4), nota-se que a taxa metabólica basal é maior $(p=0,0175)$ nos pacientes sem a Síndrome e o tempo de infecção $(p=0,0020)$ e de TARV $(p=0,0002)$ são menores. Ainda é interessante ressaltar, mesmo que não seja estatisticamente significante, que o percentual de gordura é menor $(-12,5 \%)$, a massa magra é maior $(4,9 \%)$ e a massa gorda é menor $(-9,5 \%)$ nos pacientes sem a Síndrome.

Na tabela 5 observa-se que tanto os pacientes sem como os com lipodistrofia apresentaram dois domínios abaixo do índice de 50, a preocupação financeira e com o sigilo. Também não foi notada nenhuma diferença estatística entre os domínios com a presença ou não da lipodistrofia.

Observando-se a tabela 6 pode-se notar que a medicação mais utilizada pelos pacientes de forma geral foi a Lamivudina (29,40\%), seguida pelo Efavirenz $(23,20 \%)$ e pela Zidovudina (22,70\%). Quando faz-se a separação para os pacientes que apresentam a Síndrome Lipodistrófica, o três medicamentos mais usados são os mesmos, contudo troca-se a sequência, Lamivudina (29\%), Zidovudina (24\%) e Efavirenz (23\%). Já para os pacientes que não apresentam a Síndrome Lipodistrófica a sequência foi a mesma apresentada na forma geral,
Lamivudina (30\%), Efavirenz (23\%) e Zidovudina (21,80\%). Desta forma, nota-se que esses três medicamentos correspondem por 75,30\% do total de utilização.

Quanto à utilização dos inibidores de protease, o CTA/SAE de Santarém-PA utilizou como recurso terapêutico cinco tipos, onde correspondem a 13\% das medicações administradas, sendo o Ritonavir (6\%) e Lopinavir $(5,40 \%)$ os dois mais usados.

Complementa-se que dos tipos de medicações administradas para os pacientes, 13\% são inibidores de protease, 24,80\% são os inibidores de transcriptase reversa não-análogos de nucleosídeos e 62,20\% são inibidores de transcriptase reversa análogos de nucleosídeos.

Tabela 4. Demonstrativo dos valores antropométricos, da composição corporal, força de preensão manual, tempo de infecção e terapia antirretroviral em portadores e não da Síndrome Lipodistrófica.

\begin{tabular}{c|c|c|c|c|c|c|c|c|c}
\hline & \multicolumn{5}{|c|}{ Lipodistrofia } & \multicolumn{5}{c|}{ Sem Lipodistrofia } & \\
\hline & mín & máx & média & dp & mín & máx & média & dp & p \\
\hline MC (Kg) & 43,20 & 90,60 & 63,89 & 11,19 & 39,00 & 97 & 64,55 & 11,88 & $>0,05$ \\
\hline EST (m) & 1,42 & 1,87 & 1,61 & 0,10 & 1,46 & 1,82 & 1,63 & 0,08 & $>0,05$ \\
\hline IMC (Kg/m²) & 18,02 & 35,80 & 24,57 & 3,07 & 15,82 & 36,03 & 24,25 & 4,18 & $>0,05$ \\
\hline ICQ & 0,57 & 1,08 & 0,94 & 0,09 & 0,69 & 1,05 & 0,91 & 0,08 & $>0,05$ \\
\hline LDC (mm) & 40,40 & 188,5 & 109,41 & 40,90 & 27,30 & 294,3 & 107,17 & 54,86 & $>0,05$ \\
\hline \%G & 6,3 & 45,70 & 26,57 & 8,70 & 4,4 & 50,70 & 23,23 & 9,77 & $>0,05$ \\
\hline MM (Kg) & 31,69 & 73,22 & 46,76 & 9,08 & 30,89 & 79,65 & 49,05 & 8,81 & $>0,05$ \\
\hline MG (Kg) & 2,73 & 39,30 & 17,12 & 6,69 & 1,78 & 45,02 & 15,50 & 8,40 & $>0,05$ \\
\hline TMB (kcal) & 1083 & 1978 & 1421,14 & 210,81 & 1114 & 2016 & 1507,62 & 192,75 & 0,0175 \\
\hline FMd (Kg) & 18 & 52 & 31,42 & 8,81 & 10 & 53 & 32,44 & 9,23 & $>0,05$ \\
\hline FMe (Kg) & 18 & 58 & 31,83 & 9,65 & 15 & 56 & 32,79 & 9,70 & $>0,05$ \\
\hline Tinf (meses) & 4 & 180 & 62,45 & 48,69 & 1 & 183 & 37,92 & 34,42 & 0,0020 \\
\hline $\begin{array}{c}\text { TTARV } \\
\text { (meses) }\end{array}$ & 0 & 180 & 58,57 & 49,28 & 0 & 117 & 26,77 & 27,97 & 0,0002 \\
\hline I - - & & & & & & & & \\
\hline
\end{tabular}

MC - massa corporal; EST - estatura; IMC - índice de massa corporal; ICQ - índice cintura-quadril; $\sum D C$ somatório das dobras cutâneas; \%G - percentual de gordura; MM - massa magra; MG - massa gorda; TMB - taxa metabólica basal; FMd - força muscular de apreensão da mão direita; FMe - força de apreensão da mão esquerda; Tinf - tempo de infecção pelo HIV; TTARV - tempo de terapia antirretroviral; mín - mínimo; máx - máximo; dp - desvio padrão.

Tabela 5. Demonstrativo dos valores da qualidade de vida em sedentários e ativos.

\begin{tabular}{c|c|c|c|c|c|c|c|c|c}
\hline & \multicolumn{4}{|c|}{ Sem Lipodistrofia } & \multicolumn{4}{c|}{ Com Lipodistrofia } & \\
\hline & mín & máx & média & dp & mín & máx & média & dp & p \\
\hline FG & 4 & 100 & 64,64 & 21,61 & 8 & 100 & 66,68 & 21,18 & $>0,05$ \\
\hline SV & 6 & 100 & 72,37 & 21,98 & 13 & 100 & 71,34 & 22,03 & $>0,05$ \\
\hline PS & 0 & 100 & 57,33 & 32,14 & 0 & 100 & 60,88 & 32,16 & $>0,05$ \\
\hline PF & 0 & 100 & 49 & 34,24 & 0 & 100 & 38,17 & 29,30 & $>0,05$ \\
\hline PM & 35 & 100 & 75,90 & 20,63 & 20 & 100 & 72,11 & 25,54 & $>0,05$ \\
\hline AHIV & 0 & 100 & 54,07 & 36,06 & 0 & 100 & 52 & 36,04 & $>0,05$ \\
\hline Psig & 0 & 100 & 33,77 & 27,92 & 0 & 100 & 37,56 & 29,33 & $>0,05$ \\
\hline CP & 42 & 100 & 88,74 & 17,03 & 17 & 100 & 88 & 21,65 & $>0,05$ \\
\hline FX & 0 & 100 & 75,77 & 33,31 & 0 & 100 & 62,63 & 36,72 & $>0,05$ \\
\hline
\end{tabular}

FG - função geral; SV - satisfação com a vida; PS - preocupação com a saúde, PF - preocupação financeira; PM - preocupação com a medicação; AHI - aceitação com o HIV; Psig - preocupação com o sigilo; CP - confiança no profissional; FX - função sexual; mín - mínimo; máx - máximo; dp - desvio padrão. 
Tabela 6. Demonstrativo das medicações utilizadas pelos pacientes que apresentam ou não a Síndrome Lipodistrófica.

\begin{tabular}{|c|c|c|c|c|c|c|}
\hline \multirow[b]{2}{*}{ Medicações } & \multicolumn{2}{|c|}{ Lipodistrofia } & \multicolumn{2}{|c|}{ Sem Lipodistrofia } & \multicolumn{2}{|c|}{ Geral } \\
\hline & $\mathbf{n}$ & $\%$ & $\mathbf{n}$ & $\%$ & $\mathbf{n}$ & $\%$ \\
\hline \multicolumn{7}{|c|}{ Inibidores da Transcriptase Reversa Análogos de Nucleosídeos } \\
\hline TDF & 11 & 7,5 & 21 & 8,9 & 32 & 8,3 \\
\hline 3TC & 43 & 29 & 70 & 30 & 113 & 29,4 \\
\hline DDI & 2 & 1 & 0 & 0 & 2 & 0,5 \\
\hline$A B C$ & 1 & 0,7 & 1 & 0,4 & 2 & 0,5 \\
\hline D4T & 1 & 0,7 & 2 & 0,9 & 3 & 0,8 \\
\hline AZT & 36 & 24 & 51 & 21,8 & 87 & 22,7 \\
\hline$\sum$ parcial & 94 & 62,9 & 145 & 62 & 239 & 62,2 \\
\hline \multicolumn{7}{|c|}{ Inibidores da Transcriptase Reversa Não-Análogos de Nucleosídeos } \\
\hline NVP & 2 & 1 & 4 & 1,7 & 6 & 1,6 \\
\hline EFV & 35 & 23 & 54 & 23 & 89 & 23,2 \\
\hline$\sum$ parcial & 37 & 24 & 58 & 24,7 & 95 & 24,8 \\
\hline \multicolumn{7}{|c|}{ Inibidores de Protease } \\
\hline ATV & 1 & 0,7 & 3 & 1,3 & 4 & 1 \\
\hline RTV & 8 & 5,5 & 15 & 6,4 & 23 & 6 \\
\hline IDV & 1 & 0,7 & 0 & 0 & 1 & 0,3 \\
\hline LPV & 8 & 5,5 & 13 & 5,6 & 21 & 5,4 \\
\hline NFV & 1 & 0,7 & 0 & 0 & 1 & 0,3 \\
\hline$\sum$ parcial & 19 & 13,1 & 31 & 13,3 & 50 & 13 \\
\hline TOTAL & 150 & 100 & 234 & 100 & 384 & 100 \\
\hline
\end{tabular}

TDF - Tenofovir; 3TC - Lamivudina; DDI - Didanosina; ABC - Abacavir; D4T - Estavudina; AZT - Zidovudina; NVP - Nevirapina; EFV - Efavirenz; ATV - Atazanavir; RTV - Ritonavir; IDV - Indinavir; LPV - Lopinavir - NFV - Nelfinavir.

\section{DISCUSSÃO}

Salienta-se primeiramente que o CTA/SAE de Santarém - PA atende uma região de vinte municípios e, sendo assim, 62,50\% da amostra foram de pacientes residentes no município de Santarém - PA. Além disso, a etnia predominante foi parda(68.30\%), seguida pela branca $(19,20 \%)$, preta (10\%), amarela (1,70\%) e indígena (0,80\%).

Em estudos já realizados, nota-se que os pacientes HIV/Aids são predominantemente classificados como sedentários $62 \%, 64,60 \%$ (estado do Paraná20,21) ou 61,20\% (Estado de São Paulo 22 ), o que também pôde ser notado neste estudo (70\% sedentário vs 30\% ativo).

Os pacientes ativos demonstraram de forma não estatística, mas relevantes, maiores valores para a força muscular de preensão, a massa magra e taxa metabólica basal. Além de apresentarem maior tempo de infecção pelo HIV e de utilização da TARV. Os valores maiores da massa muscular e da força muscular se fazem importantes para evitar complicações e risco de mortalidade no HIV/Aids ${ }^{23}$

No estudo de Dutra et al. ${ }^{24}$, realizado na capital do Pará, notou-se que em uma amostra de 39 pacientes com lipodistrofia, o tipo predominante foi a mista, seguida pela lipoatrofia e lipohipertrofia. No estudo apresentado, 37\% da amostra apresentou lipodistrofia e com o mesmo perfil de distribuição entre os tipos. Quantitativo elevado $(46,10 \%)$ também foi observado no estudo de Arruda Júnior et al. ${ }^{25}$ No estudo de Sonaglio et al. ${ }^{17}$, realizado com crianças e adolescentes, predominou a lipoatrofia dos membros e a lipohipertrofia abdominal. Já neste estudo notou-se que tanto a lipoatrofia facial e lipohipertrofia abdominal foram as mais presentes (56\% para cada).

Quando associou-se a Síndrome Lipodistrófica com o nível de atividade física notou-se, no presente estudo, que 66\% eram sedentários. Resultado similar foi observado no estudo de Segatto et al.26 onde 63,20\% dos que apresentavam lipodistrofia eram sedentário. Além do mais, destaca-se que o estilo de vida ativo proporciona 79\% menos chance de desenvolver a lipodistrofia, bem como, a hipertensão arteria|25.

Analisando especificadamente a presença ou não da lipodistrofia, nota-se que a Síndrome Lipodistrófica pode estar associada com o tempo de infecção e tempo de terapia antirretroviral ${ }^{26,27}$. Diante disso, o estudo em questão também notou que os pacientes com lipodistrofia apresentavam taxa metabólica basal menor e com tempo de infecção pelo HIV e uso da TARV por maior tempo $(p<0.05)$. Contudo não foi observada associação com a utilização com o tipo de TARV. Outro ponto relevante de salientar é que os pacientes com lipodistrofia demonstram maior percentual de gordura no tronco, bem como, os sedentários ${ }^{26}$. Neste estudo foi possível demonstrar, não estatisticamente, que os pacientes sedentários tinham menor massa magra que os ativos e que os com lipodistrofia tinham maior percentual de gordura, massa gorda e menor massa magra que os sem lipodistrofia.

Quanto à qualidade de vida os pacientes ativos apresentaram maiores escores para quase todos os domínios, exceção para a confiança profissional. Contudo o único domínio estatisticamente melhor no ativo foi a preocupação com o sigilo, possivelmente por conviverem mais junto a outras pessoas durante as atividades físicas ou não realizadas. A presença ou não da lipodistrofia não foi fator para potencializar ou diminuir os escores dos domínios da qualidade de vida, pois nas duas situações a preocupação financeira e a com o sigilo foram as que apresentaram menores escores, bem como, nos ativos e sedentários. Quanto à perda do sigilo, Seidl e Machado ${ }^{28}$ também notaram esses relatos em pacientes HIV com lipodistrofia. Já preocupação financeira pode-se justificar por a maioria dos pacientes estarem na renda de menos de 1 salário mínimo a 4 salários (88,30\%). Esses resultados também vão ao encontro do estudo de Reis et al. ${ }^{29}$, que observaram escores menores para os referidos domínios, bem como no estudo de Lopes et al. ${ }^{30} \mathrm{com}$ pacientes acima de 50 anos.

A maior utilização, neste estudo, dos esquemas de TARV com inibidores de transcriptase reversa análogos de nucleosídeos vai ao encontro do estudo de Diehl et al. ${ }^{27}$ e Tsuda et al. ${ }^{31}$, que tanto pacientes com lipodistrofia como sem foram os medicamentos predominantes, bem como, o esquema predominante nos estudos e neste foi o AZT/3TC/ EFV (47,30\%). Além do mais, ressalta-se a não associação da utilização de inibidores de protease com a lipodistrofia neste estudo.

Destaca-se a relevância de outros estudos acrescentarem, às informações dadas neste manuscrito, o perfil e a associação das informações imunológicas, virológicas, bioquímicas e hematológicas, para possibilitar a identificação de pontos positivos ou negativos associados às variáveis estudadas.

\section{CONCLUSÃO}

De acordo com a metodologia proposta, destaca-se que a prática de atividade física entre as pessoas que vivem com o HIV/Aids é baixa e isso pode contribuir para uma diminuição da massa magra, da força de preensão manual e da taxa metabólica basal. O que também pode ser relatado para os pacientes com lipodistrofia que demonstraram taxa metabólica basal menor, tempo de infecção pelo HIV e de TARV maior, bem como, maior percentual de gordura e massa gorda, e menor massa magra. Essas informações se complementam para a importância da orientação quanto à prática orientada do exercício físico para tentar conter os fatores negativos associados ao sedentarismo e à lipodistrofia.

Quanto aos domínios da qualidade de vida com escores menores, tanto ativos/sedentários quanto pacientes com lipodistrofia/sem lipodistrofia, pontua-se a preocupação financeira e a preocupação com o sigilo. Novamente destaca-se a importância do exercício, pois tendeu a apresentar melhores escores para quase todos os domínios da qualidade de vida.

Todos os autores declararam não haver qualquer potencial conflito de interesses referente a este artigo. 
CONTRIBUIÇÕES DOS AUTORES: LFG-e-S (0000-0002-1953-9175)* e RCS (0000-0002-5308-0265)* foram os principais contribuintes na coleta de dados e, além desses, JJSF (0000-0002-0531-0935)*auxiliou na redação do manuscrito. KSK (0000-0001-6992-3557)* e LFG-e-S avaliaram os dados da análise estatística. MBX (00000003-2706-3880)* e LFG-e-S realizaram a pesquisa bibliográfica, a revisão do manuscrito e contribuíram com o conceito intelectual do estudo. *Número ORCID (Open Researcher and Contributor ID).

\section{REFERÊNCIAS}

1. Brasil. Ministério da Saúde. Boletim Epidemiológico Aids-DST. ano VI, n.1, 27a a 52ª semanas epidemiológicas - julho a dezembro de 2008, 01a a 26a semana epidemiológica - janeiro a junho de 2009, 2010 [acesso em 2015 set 21]. Disponivel em: http://www.Aids.gov.br/sites/default/files/Boletim2010.pdf

2. Brasil. Ministério da Saúde. Boletim Epidemiológico Aids-DST, ano IX, n.1, até semana epidemiológica 26a - junho 2012, 2012 [acesso em 2015 set 21]. Disponível em: http://www.Aids.gov.br/sites/default/ files/anexos/publicacao/2012/52654/boletim_jornalistas_pdf_22172.pdf

3. Brasil. Ministério da Saúde. Boletim Epidemiológico HIV-Aids, ano II, n.1, até semana epidemiológica 26a - dezembro de 2013, 2013 [acesso em 2015 set 21]. Disponível em: http://www.Aids.gov.br/sites/ default/files/anexos/publicacao/2013/55559/_p_boletim_2013_internet_pdf_p_51315.pdf

4. Gazzaruso C, Bruno R, Garzaniti A, Giordanetti S, Fratino P, Sacchi P, et al. Hypertension among HIV patients: prevalence and relationships to insulin resistance and metabolic syndrome. J. Hypertens. 2003; 21(7):1377-82.

5. Werner ML, Pone MV, Fonseca VM, Chaves CR. Lipodystrophy syndrome and cardiovascular risk factors in children and adolescents infected with HIV/Aids receiving highly active antiretroviral therapy. J Pediatr (Rio J). 2010;86(1):27-32.

6. Souza HF, Marques DC. Benefícios do treinamento aeróbico e/ou resistido em Indivíduos HIV+: uma revisão sistemática. Rev Bras Med Esporte. 2009;15(6):467-71.

7. Gomes RD, Borges JP, Lima DB, Farinatti PT. Effects of physical exercise in the perception of life satisfaction and immunological function in HIV-infected patients: non-randomized clinical trial. Rev Bras Fisioter. 2010;14(5):390-5.

8. Yarasheski KE, Tebas P, Stanerson B, Claxton S, Marin D, Bae K, et al. Resistance exercise training hypertriglycemia in HIV-infected men treated with antiviral therapy. J Appl Physiol (1985). 2001;90(1):133-8.

9. Mendes EL, Andaki ACR, Brito CJ, Córdova C, Natali AJ, Amorim PRS, et al. Beneficial effects of physical activity in an HIV-infected woman with lipodystrophy: a case report. J Med Case Rep. 2011;5(430):1-6.

10. Souza PML, Jacob-Filho W, Santarém JM, Silva AR, Li HY, Burattini MN. Progressive resistance training in elderly HIV-positive patients: does it work? Clinics (São Paulo). 2008; 63(5):619-24.

11. Dolan SE, Frontera W, Librizzi J, Ljungquist K, Juan S, Dorman R, et al. Effects of a supervised home-based aerobic and progressive resistance training regimen in women infected with human immunodeficiency virus: a randomized trial. Arch Intern Med. 2006;166(11):1225-31.

12. Lindegaard B, Hansen T, Hvid T, Van Hall G, Plomgaard P, Ditlevsen S, et al. The effect of strength and endurance training on insulin sensitivity and fat distribution in human immunodeficiency virus-infected patients with lipodystrophy. J Clin Endocrinol Metab. 2008;93(10):3860-9.

13. Spierer DK, Demeersman RE, Kleinfeld J, Mcpherson E, Fullilove RE, Alba A, et al. Exercise training improves cardiovascular and autonomic profiles in HIV. Clin Auton Res. 2007;17(6):341-8.

14. Driscoll SD, Meininger GE, Ljungquist K, Hadigan C, Torriani M, Klibanski A, et al. Differential effects of metformin and exercise on muscle adiposity and metabolic indices in human immunodeficiency virus-infected patients. J Clin Endocrinol Metab. 2004;89(5):2171-8.

15. Engelson ES, Agin D, Kenya S, Werber-Zion G, Luty B, Albu JB, et al. Body composition and metabolic effects of a diet and exercise weight loss regimen on obese, HIV-infected women. Metabolism. 2006; 55(10):1327-36.
16. Fernandes Filho J. A prática da avaliação física. 2a.ed. Rio de Janeiro: Shape; 2003.

17. Sonaglio EP, Pedro FL, Silva QH, Kirsten VR. Síndrome da lipodistrofia em crianças e adolescentes com HIV/Aids em uso de terapia antirretroviral. Rev AMRIGS. 2011;55(3):224-8.

18. Matsudo SMM, Matsudo VKR, Barros Neto TL. Atividade física e envelhecimento aspectos epidemiológicos. Rev Bras Med Esporte. 2001;7(1):2-13.

19. Soárez PC, Castelo A, Abrão P, Holmes WC, Ciconelli RM. Tradução e validação de um questionário de avaliação de qualidade de vida em Aids no Brasil. Rev Panam Salud Publica. 2009;25(1):69-76.

20. Romancini JLH, Guariglia D, Nardo Junior N, Herold J, Pimentel GGA, Pupulin ART. Níveis de atividade física e alterações metabólicas em pessoas vivendo com HIV/Aids. Rev Bras Med Esporte 2012;18(6):356-60.

21. Guariglia DA, Avelar A, Cucato GG, Reichert FF, Cyrino ES. Atividade física habitual em portadores de HIV/Aids. Rev Educação Física/UEM. 2007;18(Supl):260-2.

22. Silva EFR, Lewi DS, Vedovato GM, Garcia VRS, Tenore SB, Bassichetto KC. Estado nutricional, clínico e padrão alimentar de pessoas vivendo com HI/Aids em assistência ambulatorial no município de São Paulo. Rev Bras Epidemiol. 2010;13(4):677-88

23. Roubenoff R, Grinspoon S, Skolnik PR, Tchetgen E, Abad L, Spiegelman D, et al. Role of cytokines and testosterone in regulating lean body mass and resting energy expenditure in HIV-infected men. Am J Physiol Endocrinol Metab. 2002;283(1):E138-45.

24. Dutra CDT, Salla LCN, Marques MCM, Libonati RMF. Avaliação do consumo alimentar em pacientes HIV positivos com lipodistrofia. Rev Ciênc Saúde. 2011;4(2):59-65.

25. Arruda Junior ER, Lacerda HR, Moura LCRV, Albuquerque MFPM, Miranda Filho DB, Diniz GTN, et al. Perfil dos pacientes com hipertensão arterial incluídos em uma coorte com HIV/Aids em Pernambuco Brasil. Arq Bras Cardiol. 2010;95(5):640-7.

26. Segatto AFM, Freitas Junior IF, Santos VR, Alves KCP, Barbosa DA, Portelinha Filho AM, et al. Lipodystrophy in HIV/Aids patients with different levels of physical activity white on antiretroviral therapy. Rev Soc Bras Med Trop. 2011:44(4):420-4

27. Diehl LA, Dias JR, Paes ACS, Thomazini MC, Garcia LR, Cinagawa E, et al. Prevalência da lipodistrofia associada ao HIV em pacientes ambulatoriais brasileiros: relação com a síndrome metabólica e fatores de risco cardiovascular. Arq Bras Endocrinol Metab. 2008;52(4):658-67.

28. Seidl EMF, Machado ACA. Bem-estar psicológico, enfrentamento e lipodistrofia em pessoas vivendo com HIV/Aids. Psicol Estudo. 2008;13(2):239-47.

29. Reis RK, Haas VJ, Santos CB, Teles AS, Galvão MTG, Gir E. Sintomas de depressão e qualidade de vida de pessoas vivendo com HIV/Aids. Rev Latino-Am Enfermagem. 2011;19(4):1-8.

30. Lopes PSD, Silva MMG, Torres IC, Stadnik CMB. Qualidade de vida dos pacientes HIV positive com mais de 50 anos. Rev AMRIGS. 2011;55(4):356-60.

31. Tsuda LC, Silva MM, Machado AA, Fernandes APM. Alterações corporais: terapia antirretroviral e síndrome da lipodistrofia em pessoas vivendo com HIV/Aids. Rev Latino-Am Enfermagem. 2012;20(5):1-7. 